\title{
Distributional Assumptions and Observed Conservatism in the Theory of Signal Detectability
}

\author{
Laurence T. Maloney \\ Department of Psychology and Department of Electrical Engineering \\ and Computer Science, University of Michigan
}

AND

EWART A. C. Thomas

Department of Psychology, Stanford University

\begin{abstract}
The theory of signal detectability typically fits data from Yes-No detection experiments by assuming a particular form for the noise and signal plus noise distributions of the Observer. Previous work suggests that estimates of the Observer's sensitivity are little affected by small discrepancies between the assumed distributions (usually Gaussian) and the Observer's true underlying distributions. Possibly for this reason, estimates of the Observer's choice of criterion or likelihood ratio suggesting suboptimal performance have also been taken at face value. It is, for example, commonly accepted that human Observers are conservative: They are said to choose criteria corresponding to likelihood ratios that are closer to 1 than the ratios produced by optimal criteria. We demonstrate that estimates of likelihood ratio can be markedly biased when the distributions assumed in estimation are not the Observer's true distributions. We derive necessary and sufficient conditions for an optimal Observer to appear conservative when fitted by distributions different from those governing his choices.

These results raise a fundamental question: What information about the Observer's underlying noise and signal plus noise distributions does the Observer's performance in a Yes-No detection task provide? We demonstrate that a small number of isosensitivity (ROC) curves completely determines the Observer's underlying noise and signal plus noise distributions for many familiar forms of the theory of signal detectability. These results open up the possibility of a semiparametric theory of signal detectability. 1991 Academic Press, Inc.
\end{abstract}

Reprint requests should be sent to Laurence $T$. Maloney, who is now at the Department of Psychology, 8th Floor, New York University, 6 Washington Place, New York, New York 10003.

This work was supported in part by an associateship awarded to the first author by the National Research Council of the National Academy of Sciences administered through the NASA Ames Research Center, Life Sciences Division, at Moffett Field, Mountain View, CA and in part by a grant to the first author from the National Eye Institute, EY08266. We are grateful for comments provided by Jean-Claude Falmagne, David H. Krantz, Donald R. J. Laming, Michael S. Landy, David E. Meyer, A. A. J. Marley, James T. Townsend, and an anonymous reviewer whose comments much improved the presentation. 


\section{INTRODUCTION}

The theory of signal detectability (TSD), in its simplest form, summarizes an Observer's decision-making under uncertainty via two parameters. One parameter, $d^{\prime}$, measures the Observer's sensitivity or capacity, and the other parameter, $\beta$, is a criterion that indexes the effect of motivational factors on the Observer's performance. Since the original presentation of TSD by Tanner and Swets (1954), various aspects of the theory have been examined, including (a) optimal statistical methods for estimating the two parameters (e.g., Altham, 1973; Dorfman, Beavers, \& Saslow, 1973; Grey \& Morgan, 1972; Ogilvie \& Creelman, 1968), (b) the effects of varying the form of the probability distributions of noise and signal plus noise (Egan, 1975; Green \& Swets, 1966/1974; Lockhart \& Murdock, 1970; Morgan, 1976; Pollack \& Hsieh, 1969; Swets, 1986a, 1986b), and (c) the effects of the subject's use of suboptimal decision rules on the parameter estimates (Creelman \& Donaldson, 1968; Thomas \& Legge, 1970).

A general conclusion from studies under (a) and (b) is that estimates of the sensitivity parameter are quite robust under various distributional assumptions (e.g., Morgan, 1976). Possibly for this reason, estimates of the Observer's criterion or likelihood ratio have also been taken at face value. These estimates, generally obtained under the assumption of Gaussian equal-variance distributions of noise and signal plus noise, constitute the focus of many studies under (c). The estimated criterion is often found to be less extreme than the value that is optimal for the decision, a type of suboptimality that has been labeled "conservatism". One of the issues considered in the present article is the robustness of such conservatism under various distributional assumptions.

Human judgment and decision-making have been found to be suboptimal in a variety of contexts. While these findings are important as descriptions of behavior, they also provide the impetus for studies of the nature and causes of suboptimal behavior. For example, studies showing that judgments of event likelihood sometimes violate the axioms of probability theory (Kahneman, Slovic, \& Tversky, 1982) are invoked to explain why judgments between complex gambles can be suboptimal.

Other studies attempt to account for suboptimality by identifying a rule or heuristic to which the data seem to conform. In the case of conservatism, suboptimality has been attributed to (a) a decision-making heuristic that fails to maximize expected payoff, or (b) an unstable criterion. Much attention has been given to probability matching as a heuristic leading to conservatism (Creelman \& Donaldson, 1968; Parks, 1966; Thomas \& Legge, 1970). Or, if the Observer varies the criterion from trial to trial but the data is fitted with the assumption that the criterion is fixed, the estimated critcrion will differ from the optimal for many choices of sensory distributions. Attention has centered on whether there are plausible rules for this trial-by-trial variation such that the Observer's overall data conform to probability matching or some other heuristic. (See, for example, Dusoir (1974) and Thomas (1975) for discussions of the adequacy of the probability matching heuristic and other rules for criterion adjustment.) 
In all these discussions, it is assumed that the experimenter knows the sensory distributions of the Observer. Laming (1986), however, has raised doubts concerning the use of Gaussian distributions for certain kinds of tasks. Studies of detectability, hased on stimulus energy (or amplitude) in vision and audition lead to a variety of distribution forms: non-central $\chi^{2}$, non-central $F$, and others. While Swets (1986b) finds that Gaussian TSD fits data from several different kinds of discriminative and diagnostic tasks well, at least onc of his data sets seems to exhibit patterned deviations from the Gaussian model (his Fig. 6c, d).

If the distributional assumptions underlying data reduction are incorrect;-it is possible to construct another account of observed suboptimal behavior. According to this alternative account, behavior might be optimal in reality but appear to be suboptimal only because the experimenter has incomplete information about the Observer. Such an account, originally suggested by Laming (1973, p. 95), would be a corrective to the prevailing view that human Observers are far from ideal, even in simple perceptual tasks (compare Healy \& Kubovy, 1981; Kubovy, 1977).

In this article, we present two sets of mathematical results concerning the distributional assumptions commonly made in fitting empirical data via TSD. We first characterize precisely how choice of distribution influences parameter estimation for a broad class of TSD models. These results permit us to state necessary and sufficient conditions for an optimal Observer erroneously to be labeled conservative as a consequence of incorrect assumptions concerning the Observer's underlying distributions.

Second, we demonstrate that much weaker distributional assumptions than those commonly used suffice, in principle, to permit estimation of the Observer's sensitivity and criterion. We do so by characterizing what information about the Observer is available in the Observer's performance summarized as a small number of isosensitivy (ROC) curves.

In combination our results indicate that conventional distributional assumptions made in fitting Observer's data in signal detection tasks are (a) potential sources of error, and (b) unnecessary.

\section{The TSD Observer: DeFinitions}

The TSD Model. TSD provides a model of the Observer's signal detection behavior based on two assumptions. The first is that the sensory information available to the TSD Observer is a random variable $X$. On trials where the signal is absent, $X$ has the cumulative distribution function (cdf) $F_{\mathrm{N}}(x)$, called the noise distribution. On trials where the signal is present, $X$ has the cdf $F_{\mathrm{SN}}(x)$, the signal plus noise distribution. These two distributions are the sensory distributions. When the corresponding probability density functions (pdf's) exist, they will be denoted $f_{\mathrm{N}}$ and $f_{\mathrm{SN}}$, respectively.

The second assumption is that the Observer has a decision rule used to decide whether noise alone or signal plus noise was present on a given trial. A decision 
rule is a function $D(X)$ that specifies "Yes" or "No" for each value of $X$. For example (iff is an abbreviation for "if and only if"),

$$
D(X, c)=\mathrm{YES} \quad \text { iff } \quad X>c
$$

is a decision rule based on a sensory criterion: The Observer establishes the sensory criterion, $c$, and responds Yes (signal) or No (no signal) accordingly as the sensory information $X$ is greater than or less than the criterion. Each possible choice of $F_{\mathrm{N}}, F_{\mathrm{SN}}$, and $D$ is a candidate model for a TSD Observer. We will sometimes refer to such a model as a "TSD model," or as a "TSD," or as a "model Observer."

The goal of estimation is to identify the empirical Observer, to match the Observer's behavior to a specific model Observer. This goal is not achievable without drastic restrictions on the possible distributions $F_{\mathrm{N}}, F_{\mathrm{SN}}$ (see Laming, 1973, pp. 100-101; Marley, 1971). The conventional approach to estimation is to assume that we know the Observer's distributions except for a small number of parameter values. $F_{\mathrm{N}}$ and $F_{\mathrm{SN}}$ are commonly assumed to be Gaussian distributions with the same standard deviation $\sigma$ and with means at 0 and $d$, respectively (See Fig. 1).

Location TSDs and S3. We next define two subsets of all the possible TSD model Observers which we refer to as location TSDs and S3. Both classes are natural generalizations of familiar equal-variance Gaussian TSD where the Gaussian is replaced by some other distribution. S3 is a subset of the location TSDs which we use to explore the distributional robustness of TSD in the first part of this paper. We use the broader class of location TSDs when we determine what information about the Observer is contained in the Observer's behavior in a signal detection task.

Definition 2.1. A TSD model is a location TSD if and only if $F_{\mathrm{SN}}(x)=$ $F_{\mathrm{N}}(x-d), d \geqslant 0$ : the signal plus noise distribution is a copy of the noise distribution shifted by $d$.

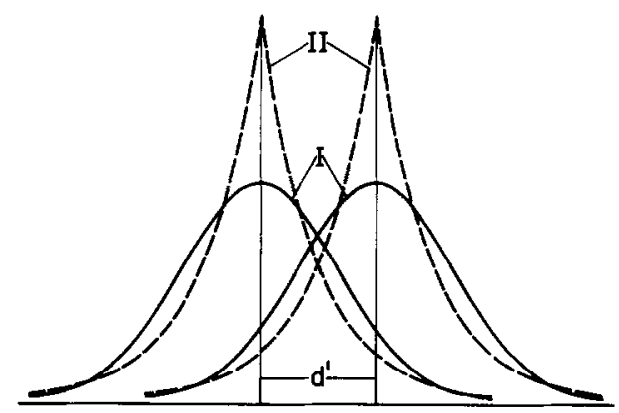

FIG. 1. The sensory distributions for two location TSDs, the first based on the Gaussian (solid lines), the second based on the Laplacian (dashed lines). 
As noted above, Fig. 1 shows the pdf's of the sensory distributions for two examples of location TSDs. The first is equal-variance Gaussian shown in solid lines. The second is similar, but the Gaussian distribution has been replaced by the Laplacian distribution shown in dashed outline. The sensory distributions of a lociation TSD are always identical except that $F_{\mathrm{SN}}$ is shifted by a distance $d$. To specify a location TSD we need specify $F_{\mathrm{N}}$, the parameter $d$, and the parameters specifying the decision rule. In the case of the sensory criterion rule above, there is a single parameter $c$. A location TSD with a sensory criterion is completely specified by $F_{\mathrm{N}}, d$, and $c$.

For convenience, we shall usually assume that the noise distribution $F_{\mathrm{N}}$ is normalized so that the median of $F_{\mathrm{N}}$ is at 0 , and the upper quartile (75th percentile) of $F_{\mathrm{N}}$ is at 1 . This normalization is the analogue of using the unit-variance, zero mean Gaussian as the noise distribution in fitting equal-variance Gaussian TSD. Since at least one of the distributions that interests us does not have a finite mean or variance, we normalize to the median and upper quartile. Also, for convenience, we sometimes restrict attention to those cdf's that have corresponding pdf's. This assumption reduces the size of the class of location TSDs, but not in any empirically significant way, since any cdf without a pdf can be approximated arbitrarily closely by cdf's that do have pdf's.

Next we define S3. S3 is a subset of the location TSDs chosen because of its convenient mathematical properties. The assumptions made in delimiting this class are technical in nature and some of them could easily be dropped without affecting the conclusions drawn. However, the results of the next section would not be appreciably strengthened by increasing the size of S3, and doing so would reduce the readability of the proofs offered.

DefinITION 2.2. A TSD model is in $\mathbf{S 3}$ if it is a location TSD whose sensory distributions further satisfy

(a) the cdf, $F(x)$, can be differentiated (except possibly at a finite number of points) to yield a probability density function $f(x)$ that is unimodal (has a single maximum), and

(b) the pdf's $f_{\mathrm{N}}(x) \equiv f(x)$ and $f_{\mathrm{SN}}(x) \equiv f(x-d)$ have support everywhere; i.e., $f_{\mathrm{N}}(x)>0$, and $f_{\mathrm{SN}}(x)>0$, for all $x$.

(c) the pdf, $f(x)$, is symmetric about its median.

The initial letters of the three conditions (Support, Smoothness, Symmetry) give us the notation S3. Only the last assumption, symmetry, and the assumption of unimodality, are empirically significant restrictions. Any locations TSD can be arbitrarily well approximated by location TSDs that are smooth and have support everywhere. We speak of model Observers (or TSDs) as being in S3 and we will also refer to distributions as being in S3. The Gaussian and Laplace distributions (Fig. 1) are members of S3.

Figure 2 summarizes the classes of TSD models we use. In addition to S3 and the location TSDs, we have diagrammed the class of location-scale TSDs which we 
All TSDs

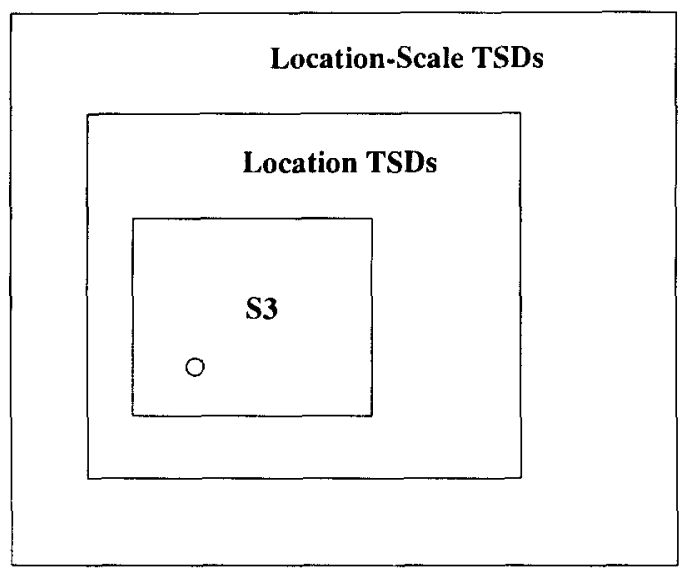

FIG. 2. The classes of model TSD Observers discussed. The small circle represents the equal-variance Gaussian TSD Observer.

touch on briefly in a later section. They are model TSDs that generalize Gaussian TSD where the assumption of equal variance has been relaxed.

The Sensory Criterion Decision Rule. If the distributions of a TSD are smooth enough to have probability density functions, we may define the likelihood ratio $\beta$ corresponding to a given sensory criterion $c$ to be $L(c)=f_{\mathrm{SN}}(c) / f_{\mathrm{N}}(c)$. For a location TSD, this simplifies to $L(c)=f(c-d) / f(c)$ where $f=f_{\mathrm{N}}$. For many location TSDs, including the Gaussian, the likelihood ratio, $\beta$, is a monotonic function of $c$. For this class, the $x$-axis is monotonic with the likelihood ratio axis and the isosensitivity curve is concave everywhere. Our Observer, who uses the $x$-axis as the decision axis, would then be the ideal Observer of TSD for whom the decision axis is the likelihood ratio axis.

Throughout the analyses following we assume that the model Observer uses a sensory criterion rule, $X>c$. This assumption accords with observed human performance in signal detection tasks. When the TSD has the monotone likelihood ratio property (that the likelihood ratio $\beta$ is a monotone increasing function of the sensory criterion $c$ ), this rule coincides with the likelihood criterion rule $L(X)>\beta$. Because of our interest in the biasing effects of distributional assumptions, we did not want to restrict the location TSDs considered to have the monotone likelihood ratio property. We have chosen instead to consider, at least initially, distributions "resembling" the Gaussian, in the sense that the corresponding isosensitivity curves are symmetric about the negative diagonal and are concave in the middle region where most task outcomes lie. These are the distributions that can be distinguished from the Gaussian family only with much difficulty. They include some, like the Cauchy family, that are not monotone likelihood ratio families. All of the proofs 
below are valid when we restrict ourselves to the members of $\mathbf{S 3}$ and location TSDs exhibiting the monotone likelihood ratio property.

The Isosensitivity Curve. Last, we define the isosensitivity curve (receiver operating characteristic) representing the part of the TSD model that is observable empirically. The outcome of a Yes-No task is usually characterized by a false alarm rate, $p_{\mathrm{r} \cdot \mathrm{A}}$, and a hit rate, $p_{\mathrm{H}}$, determined by equations $p_{\mathrm{rA}}=1-F_{\mathrm{N}}(c)$, and $p_{\mathrm{H}}=$ $1-F_{\mathrm{SN}}(c)$. If the Observer is induced to vary $c$, with $d$ held constant, across experimental conditions (say, by varying the payoffs for responses), the point $\left(p_{\mathrm{FA}}, p_{\mathrm{H}}\right)$ traces out the isosensitivity curve introduced above.

Conservatism. For any combination of payoffs and prior odds on the presence or absence of the signal, there is an optimal value of $\beta$ maximizing payoffs to the Observer. This value can be computed without knowledge of the sensory distributions. An optimal Observer will select a point along his or her isosensitivity curve corresponding to this optimal $\beta$. A conservative Observer shows a characteristic departure from optimal $\beta$ :

Definition 2.3. An Observer is conservative iff he or she consistently chooses values of $\beta$ closer to 1 than the optimal value of $\beta$ for the given payoffs and prior odds.

Green \& Swets (1966/1974) exhibit data where optimal likelihood ratio ranges from $1 / 9$ to $9 / 1$ yet Observers' estimated $\beta_{e}$ 's range only from $1 / 2.20$ to $2.17 / 1$. Green \& Swets conclude that: "[T]he observer tends to avoid extreme criteria: when the optimal $\beta$ is relatively large, his actual criterion is not so high as the optimal criterion, and when the optimal $\beta$ is relatively small, his criterion is not so low as the optimal criterion (p. 91)."

We introduce the notion of a radical Observer as well:

Definition 2.4. An Observer is radical iff he or she consistently chooses values of $\beta$ that are further from 1 than the optimal value of $\beta$ for the given payoffs and prior odds.

Note that the conservative, radical, and optimal Observers all coincide in the special case where the optimal value of $\beta$ is in fact 1 .

\section{Conservatism: An Example}

Overview. Can an experimenter's assumptions concerning the Observer's distributions lead him or her incorrectly to label an optimal Observer conservative? In this section, we demonstrate, by example, that failure to recognize the Observer's 
actual distributions can produce biased estimates of $\beta$. Apparent deviations from optimal performance are large, and very similar to the deviations exhibited by human Observers.

Suppose that the noise and signal plus noise distributions that are used by the Observer are $f_{o}(x)$ and $f_{o}\left(x-d_{o}\right)$, respectively, and that the Observer's sensory criterion is $c_{o}$, with corresponding likelihood ratio $\beta_{o}=f_{o}\left(c_{o}-d_{o}\right) / f_{o}\left(c_{o}\right)$. An experimenter wishes to use the Observer's false alarm and hit rates to estimate $d_{o}$ and $\beta_{o}$ and, for this purpose, assumes that $f_{e}(x)$ is the Observer's noise distribution. The triple, $\left[p_{\mathrm{FA}}, p_{\mathrm{H}}, f_{e}(\cdot)\right]$, would then yield estimates, $d_{\epsilon}$ and $\beta_{e}$, of $d_{o}$ and $\beta_{o}$, respectively. If $f_{e}(\cdot)=f_{o}(\cdot)$, then the experimenter's estimates would be accurate. If, however, $f_{e}(\cdot) \neq f_{o}(\cdot)$, the question arises as to whether there may be systematic deviations of $\beta_{e}$ and $d_{e}$ from $\beta_{0}$ and $d_{o}$, respectively. In the next section, we offer a complete characterization of the patterns of failures to be expected. In this section, we demonstrate, by example, that such systematic deviations can produce the appearance of conservatism in $\beta_{e}$ when the Observer is in fact choosing optimal $\beta_{o}$.

Assume that the Observer's true noise distribution is the Laplace distribution, $f_{o}(x)=\frac{1}{2} e^{-|x|}$ (Johnson \& Kotz, 1970, Chap. 23), but that the distribution, $f_{e}(x)$, used in fitting the data is the familiar, unit-variance Gaussian distribution. Let us assume also that the signal plus noise distribution (for both the true distribution and the distribution used in fitting) differs from the corresponding noise distribution by only a location transformation. Figure 1 contrast the Observer's actual distributions and the experimenter's assumptions concerning the Observer. We do not intend to recommend the use of a TSD based on the Laplacian distribution. The latter is instead a device for illustrating how estimates of the criterion parameter $c_{o}$ and likelihood ratio $\beta_{o}$ can be affected by choice of distribution. Such demonstrations motivate the results of the next section where we derive necessary and sufficient conditions that an incorrect choice of distribution mimic conservative behavior.

For a given value of $d_{o}$, say $d_{o}=1$, each choice of $c_{0}$ generates values for $p_{\mathrm{FA}}$ and $p_{\mathbf{H}}$. These values, in turn, can be inserted into the formulae for the Gaussian, unit-variance model yielding estimates, $\beta_{e}$ and $d_{e}$ (usually called $d^{\prime}$ ).

Figures $3 \mathrm{a}$ and $3 \mathrm{c}$ show plots on logarithmic axes of the estimated criterion, $\beta_{e}$, versus the true criterion, $\beta_{o}$, for $d_{o}=1$ and 3 . (Figures $3 \mathrm{e}$ and $3 \mathrm{f}$ are discussed in a later section.) The estimates, $\beta_{e}$, lie above the $45^{\circ}$ line for $\beta_{o}<1$ and below it for $\beta_{o}>1$. Estimates depart less from 1 than the true criterion values, a pattern indicating conservatism if the true values are optimal. Compare Fig. 4-3 in Green \& Swets $(1966 / 1974$, p. 92$)$.

It is interesting to note that, in an experimental situation, it would be difficult to determine that the Laplacian and not the Gaussian is the true underlying distribution. Figures $3 \mathrm{~b}$ and $3 \mathrm{~d}$ plot the residual estimated location parameter (the normalized difference between each $d_{e}$ and the mean $d_{e}$ ) versus true $\log \beta_{o}$. Note that, in comparision with the magnitude of the mean $d_{e}$, the estimates vary only slightly, though systematically, as the value of $\beta_{o}$ varies. It would be difficult to reject Gaussian theory TSD on the grounds that the estimated separation parameter varies with the choice of $\beta_{o}$. 

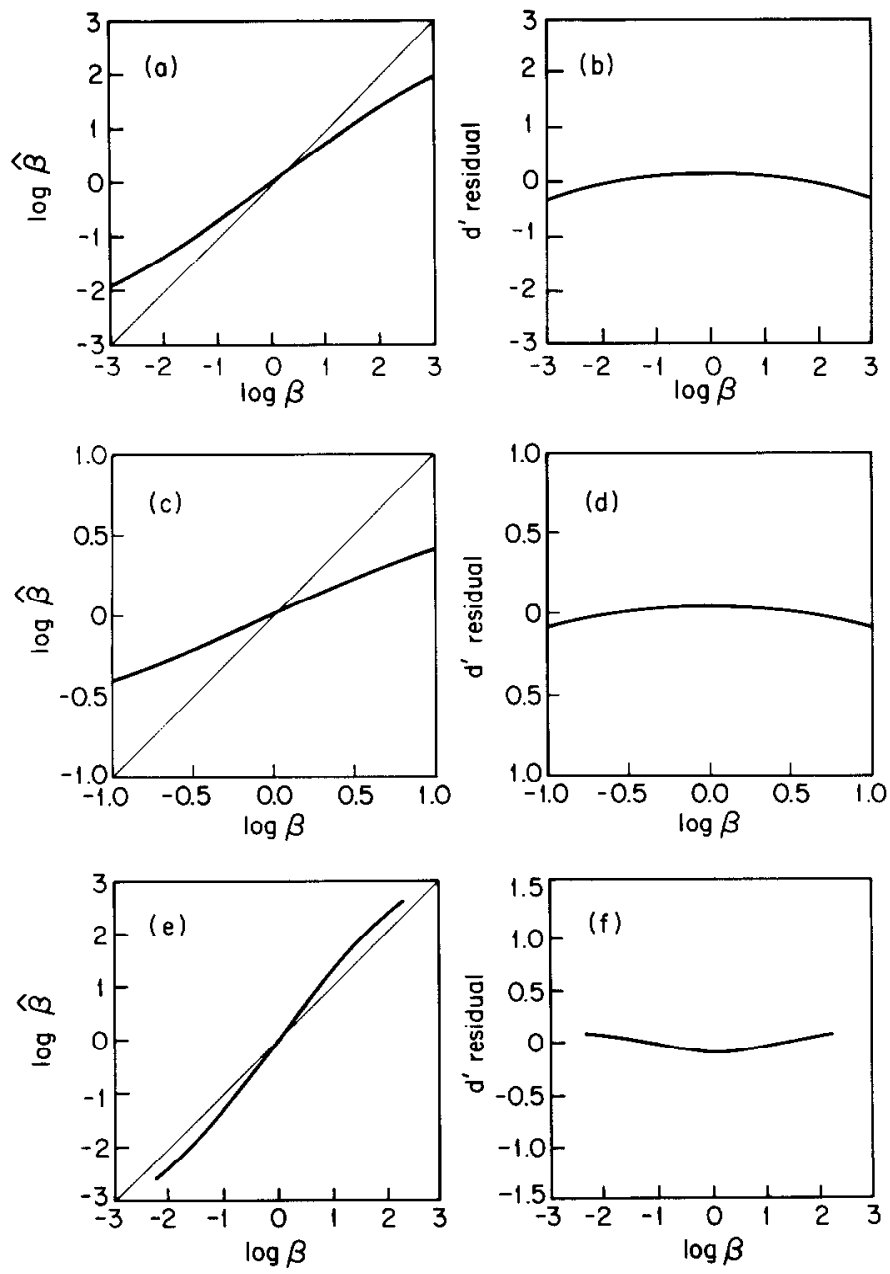

Fig. 3. (a) and (c): Plots of $\log \beta_{e}$ estimated vs true $\log \beta_{o}$ for $d_{o}=1$ and 3 , with true distribution the Laplacian. (b) and (d): Plots of residual $d_{e}$ estimated vs true $\log \beta_{o}$ for the same two distributions as in (a) and (c). (e) and (f): The same as (a) and (b), except that the true distribution is the hypernormal. The distribution assumed in fitting is always the Gaussian.

Figure 4 plots $p_{\mathrm{FA}}$ versus $p_{\mathrm{H}}$ as isosensitivity curves when $f_{o}$ is Gaussian, Laplace, and Cauchy. They are fairly similar across the middle range of outcome values, and the shape of the contour provides few clues as to which of them best describes experimental data. Swets (1986a) makes a similar point concerning the isosensitivity curves of Gaussian TSD and TSDs based on other distributions.

DEFINITION 3.1. We call the distribution $f_{o}$ locally conservative with respect to the distribution $f_{e}$ if Observers having the first distribution appear conservative for particular values of $d_{o}$ and criterion $c_{o}$ when responses are fit using $f_{e}$. 


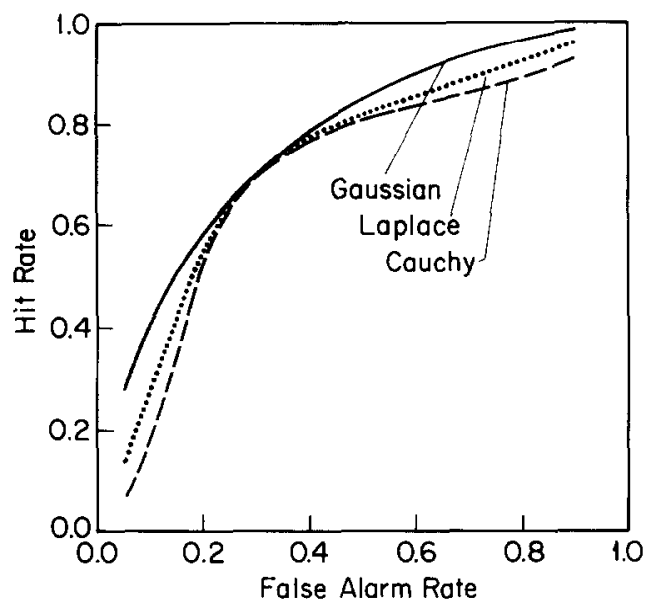

FIG. 4. Isosensitivity (ROC) curves for Gaussian, Laplace, and Cauchy location TSD models, constructed to pass through $(0.3,0.7)$ : for the Gaussian, $d=1.048$, for the Laplace, $d=1.022$, and for the Cauchy, $d=1.453$.

DefinITION 3.2. We call the distribution $f_{o}$ everywhere conservative with respect to the distribution $f_{e}$ if Observers having the first distribution appear conservative for all values of $d_{o}$ and criterion $c_{o}$ when responses are fit using $f_{\rho}$. The meaning of the term conservative used without qualification should be clear from context as in " $f_{o}$ is conservative with respect to $f_{e}$ when $d_{o}$ is greater than 2."

In the next section, we derive necessary and sufficient conditions that a distribution $f_{o}$ be conservative with respect to a second distribution $f_{e}$ either over the entire real line or any specified subinterval. We restrict attention to location TSDs with distributions drawn from $\mathbf{S 3}$ for the duration of the following section.

\section{Everywhere Conservative Relations}

Overview. In this section we introduce the quantile map $\psi$ and demonstrate that it completely characterizes biases introduced by the fitting process when the distribution assumed is not the Observer's true distribution. In particular, we state in terms of $\psi$ the condition under which an optimal Observer will erroneously be judged conservative, as in the example of the last section. We demonstrate that the results of the last section are not a special property of the Laplacian equal-variance TSD. In fact, the results of this section permit us to "manufacture," using $\psi$, an indefinitely large number of model Observers who behave normatively and optimally in actuality, but who will be judged conservative by an experimenter assuming the Gaussian equal-variance Observer. The same construction allows us to generate Observers exhibiting radical (anti-conservative) behavior. 
The conclusion we draw is that estimates of likelihood $\beta$ and, to a lesser extent, estimates of sensitivity $d$, are not distributionally robust. Without knowledge of the Observer's true distributions, estimates of $\beta$ should not be used to label an Observer conservative.

Throughout this section, we assume that all distributions are drawn from S3, as defined above. We intend to show that, within the class S3, there exist model Observers that will be diagnosed as behaving conservatively when in fact they are in fact behaving optimally. If we were to expand the class of model Observers to all location TSDs or even larger classes of TSDs, this problem of misdiagnosis would only worsen. Consequently, it suffices to develop the results below in the constricted, but mathematically tractable, class $\mathbf{S 3}$.

Definition 4.1. Define $\psi=F_{e}^{-1}{ }_{0} F_{o}$ to be the quantile map between $F_{o}$ and $F_{e}$.

Let $f_{*}$ refer to either $f_{o}$ or $f_{e}$, and let us adopt a similar notation for other quantities. Then since we are working in $\mathbf{S 3}, p_{\mathrm{FA}}=1-F_{*}\left(c_{*}\right)$ and $p_{\mathrm{H}}=1-F_{*}\left(c_{*}-d_{*}\right)$. Therefore, $F_{e}\left(c_{e}\right)=F_{o}\left(c_{o}\right) . F_{e}$ is invertible (it is a cdf in $\mathbf{S 3}$ ). Hence we have

$$
c_{e}=F_{e}^{-1} \circ F_{o}\left(c_{o}\right)=\psi\left(c_{o}\right) \text {. }
$$

A similar argument gives us

$$
c_{e}-d_{e}=F_{e}^{-1} \circ F_{o}\left(c_{o}-d_{o}\right)=\psi\left(c_{o}-d_{o}\right),
$$

expressing $c_{e}$ and $d_{e}$ as functions of the true parameters of the Observer.

The map $\psi$, the quantile map, is readily interpretable. It maps the quantiles of the distribution $f_{o}$ to the corresponding quantiles of the distribution $f_{e}$. The median of $f_{o}$ is mapped to the median of $f_{e}$, and so forth. ( $Q-Q$ plots, used to test whether a given sample of data is drawn from a hypothesized distribution, are related to the quantile map. See Gnanadesikan, 1977, pp. 198-199). For example, the map $\psi(x)=a x+b$ transforms a distribution $f_{o}$ to $f_{e}$, which is just a shifted, scaled copy of itself. Since we required above that all noise distributions be normalized to have median 0 and upper quartile 1 , we can easily show

Proposition 4.2. If $\psi$ is a linear quantile map between normalized distributions, then $\psi(x)=x$.

Proof. Substitute $\psi(0)=0$ and $\psi(1)=1$ in $\psi(x)=a x+b$.

That is, the only linear map $\psi$ betwecn normalized distributions is the identity $x$. Nex we summarize some properties of $\psi$ :

Proposition 4.3. Suppose that $F_{o}$ and $F_{e}$ are both drawn from S3. Assume both distributions to be normalized with medians at 0 and upper quartiles at 1 . Let $\psi=F_{e}^{-1} \circ F_{o}$. Then 
(a) $\psi$ is continuous.

(b) $\psi$ is strictly increasing (i.e., $\psi^{\prime}>0$ ), and therefore invertible.

(c) $\psi$ is an odd function: $\psi(-x)=-\psi(x) ; \psi(0)=0$.

(d) $\psi^{\prime}(x)=f_{o}(x) / f_{e} \circ \psi(x)$

(e) $\psi^{\prime}$ is an even function: $\psi^{\prime}(x)=\psi^{\prime}(-x)$.

Proof. (a) We defined S3 so that $F_{0}$ and $F_{e}$ are differentiable and therefore continuous. Their composition, $\psi$, is also continuous.

(b) $F_{o}$ and $F_{e}$ are both strictly increasing because the corresponding pdf's have support everywhere. As $F_{e}$ is continuous and strictly increasing, so is $F_{e}^{-1}$. Then $\psi$ is the composition of strictly incrcasing functions and therefore also strictly increasing. write

(c) The symmetry assumption (evenness) in the definition of S3 permits us to

$$
\psi(-x)=F_{e}^{-1} \circ F_{o}(-x)=F_{e}^{-1}\left(1-F_{o}(x)\right)=-F_{e}^{-1}\left(F_{o}(x)\right)=-\psi(x)
$$

(d) This result follows from differentiation of $\psi$ using the chain rule and inverse function theorem (See Apostol, 1967, pp. 174-176, 252-253).

(e) Apply the chain rule to $\psi(-x)$ to get $(d / d x) \psi(-x)=-\psi^{\prime}(-x)$. Then differentiate both sides of (c) to get $(d / d x) \psi(-x)=-\psi^{\prime}(x)$. Equate the two expressions.

Next we express conservatism in terms of $\psi$. The likelihood ratio is

$$
\beta_{*}=\frac{f_{*}\left(c_{*}-d_{*}\right)}{f_{*}\left(c_{*}\right)} .
$$

We have the following series of equivalences:

$$
\beta_{e}<\beta_{o}
$$

iff

$$
\frac{f_{e}\left(c_{e}-d_{e}\right)}{f_{o}\left(c_{o}-d_{o}\right)}<\frac{f_{e}\left(c_{e}\right)}{f_{o}\left(c_{o}\right)}
$$

iff substituting from Eqs. (1) to (2),

$$
\theta\left(c_{o}-d_{o}\right)<\theta\left(c_{o}\right)
$$

where

$$
\theta(x)=\frac{f_{e^{\circ} \psi(x)}}{f_{o}(x)}=\frac{1}{\psi^{\prime}(x)}
$$


Equation (7) is a necessary and sufficient condition that $\beta_{e}<\beta_{o}$. Rewriting Eq. (7) in terms of $\psi^{\prime}$ (recalling that $\psi^{\prime}>0$ ),

$$
\beta_{e}<\beta_{o} \quad \text { iff } \quad \psi^{\prime}\left(c_{o}\right)<\psi^{\prime}\left(c_{o}-d_{o}\right)
$$

Because of the iff condition in Eq. (8), our argument also gives necessary and sufficient conditions that $\beta_{e}=\beta_{o}$ and that $\beta_{e}<\beta_{o}$; i.e., the series of iff's (Eqs. (5)-(8)) above go through with the inequalities reversed (or replaced by equalities). Consequently, we have proven

Proposition 4.4. For TSDs drawn from S3, the quantile map $\psi$ determines the relation between true and estimated $\beta$.

We next formulate conservatism in terms of $\psi$.

Since the pdf's $f_{*}$ drawn from $\mathbf{S 3}$ are unimodal and even, we can prove

LEMMA 4.5 .

$$
\beta_{*}>1 \quad \text { iff } \quad c_{*}>\frac{d_{*}}{2}
$$

Proof. (We suppress the subscript * in the subsequent proof for clarity's sake.) Since each of the noise and signal plus noise pdf's, $f(x)$ and $f(x-d)$, has a single mode at 0 and $d$, respectively, the difference $f(x)-f(x-d)$ is positive when $x=0$, and negative when $x=d$. Therefore, since $f$ is continuous, there is a value $x_{0}$, with $0<x_{0}<d$, such that $f\left(x_{0}\right)=f\left(x_{0}-d\right)$. Therefore, $\beta=1$ when $c=x_{0}$. We now show that $\beta \geqslant 1$ iff $c \geqslant x_{0}$.

By the unimodality of $f$, for $x_{0}<c<x_{0}+d, f(c-d)>f\left(x_{0}\right)>f(c)$, which implies that $\beta>1$. For $c>x_{0}+d, c-d>x_{0}>0$, and $f(c-d)>f(c)$, again implying that $\beta>1$. A similar argument shows that $\beta<1$ if $c<x_{0}$. This concludes the proof that $\beta \geqslant 1$ iff $c \geqslant x_{0}$. Since $f$ is even, $x_{0}=d / 2$.

The definition of local conservatism for a particular $c_{o}$ and $d_{o}$ can be restated as

$$
\beta_{e}<\beta_{o} \quad \text { iff } \quad c_{o}>\frac{d_{o}}{2}
$$

Recall that $\psi$ is strictly increasing and, therefore, $\psi^{\prime}>0$.

Merging Eqs. (8) and (10), we get

$$
\psi^{\prime}\left(c_{o}\right)<\psi^{\prime}\left(c_{o}-d_{o}\right) \quad \text { iff } \quad c_{o}>\frac{d_{o}}{2} .
$$

Equation (10) is a necessary and sufficient condition for local conservatism framed only in terms of $\psi, c_{o}$, and $d_{o}$. In particular, local conservatism depends on the underlying distributions $f_{*}$ only through the quantile mapping $\psi$. 
The following theorem gives a necessary and sufficient condition on $\psi$ guaranteeing that $f_{o}$ be everywhere conservative with respect to $f_{e}$. Before turning to the theorem, however, we rewrite Eq. (10) in a more convenient form. Making the substitutions $x=c_{o}-d_{o} / 2$ and $\Delta=d_{o} / 2$, we have the following characterization of local conservatism:

$$
\psi^{\prime}(x+\Delta)<\psi^{\prime}(x-\Delta) \quad \text { iff } \quad x>0 .
$$

Note that $\Delta>0$ always since $d_{o}>0$. Therefore, $f_{o}$ will be everywhere conservative with respect to $f_{e}$ precisely when Eq. (11) holds for all $x$ and all $\Delta>0$.

Begin with a brief lemma that says, in effect, a smooth non-increasing function whose derivative is non-zero almost everywhere is actually a strictly decreasing function. This result is a technical result needed to state the subsequent theorem in its strongest possible form.

Lemma 4.6. Let $h: \mathbf{R} \rightarrow \mathbf{R}$ be a continuous, differentiable function and let $A$ be an open interval of $\mathbf{R}$. Suppose that, for $x \in A, h^{\prime}(x) \leqslant 0$ with $h^{\prime}(x)=0$ on a subset of $A$ with Lebesgue measure 0. Then $x, y \in A$ with $x<y$ implies $h(x)>h(y)$.

Proof. If $h(x)<h(y)$ then, by the mean value theorem, there is $x<\zeta<y$ with $h^{\prime}(\zeta)=(h(y)-h(x)) /(y-x)>0$ contrary to the given. So $h(x) \geqslant h(y)$. Suppose $h(x)=h(y)$. Then $h(x)=h(\zeta)=h(y)$ for any $\zeta \in(x, y)$. Consequently $h^{\prime}(\zeta)=0$ for $\zeta \in(x, y)$. But then $h^{\prime}$ is zero across an open interval $(x, y)$, a set of positive Lebesgue measure. Contradiction. Therefore, $h(x)>h(y)$.

THEOREM 4.7. Assume that the quantile mapping, $\psi$, is twice differentiable, i.e., that $\psi^{(2)}$ exists everywhere. Then $f_{o}$ is everywhere conservative with respect to $f_{e}$ iff $\psi^{(2)}(x) \leqslant 0$ for $x>0$, with equality occurring on a set of Lebesgue measure 0 .

Proof. (The second derivative of $\psi$ need not be negative everywhere in order to be conservative. If $\psi^{(2)}$ is negative except at a few points where it takes on the value 0 , conservatism still holds.) Assume, first, that $\psi^{(2)}(x) \leqslant 0$ for $x>0$, with equality occurring on a set of Lebesgue measure 0 . We show that $f_{0}$ is everywhere conservative with respect to $f_{c}$ by showing that Eq. (11) holds for all values of $\Delta$ and $x$.

Recall that since $f_{*}$ is an even function, $\psi$ is odd, $\psi^{\prime}$ is even, and $\psi^{(2)}$ is odd. Therefore, $\psi^{(2)}(x) \geqslant 0$ for $x<0$ iff $\psi^{(2)}(x) \leqslant 0$ for $x>0$. It suffices to derive the condition of Eq. (11) for $x>0$. (As mentioned above, $\Delta$ is positive.) Then, for any $x>0, \Delta>0$, we wish to show that Eq. (11) holds. We distinguish two cases:

(a) $0<x<\Delta$. Then $0<\Delta-x<\Delta$ and

$$
\psi^{\prime}(x-\Delta)=\psi^{\prime}(\Delta-x)>\psi^{\prime}(\Delta)>\psi^{\prime}(x+\Delta),
$$

the last two inequalities following from two applications of the lemma with $\psi^{\prime}$ replacing $h$.

(b) $0<\Delta<x$. Then $x-\Delta>0$ and $\psi^{\prime}(x-\Delta)>\psi^{\prime}(x+\Delta)$ by the lemma. 
We have shown that $\psi^{(2)}(x) \leqslant 0$ for $x>0$, with equality only on a set of measure 0 , implies that $f_{0}$ is everywhere apparently conservative with respect to $f_{e}$. Next we prove the converse.

If $f_{o}$ is apparcntly conscrvative with respect to $f_{e}$, then Eq. (11) is valid for any $x$ and $\Delta>0$, and it can be shown that $\psi^{(2)}(x) \leqslant 0$ for $x>0$ by letting $\Delta \rightarrow 0$. We need only shown that $\psi^{(2)}$ is not zero across some open interval. But then $\psi^{\prime}$ would be constant across an open interval, which contradicts Eq. (11). This proves the converse.

A simple consequence of Theorem 4.7 is

COROLlaRY 4.8. Assume that the quantile mapping, $\psi$, is twice differentiable, i.e., that $\psi^{(2)}$ exists everywhere. Then if $\psi^{(2)}(x)<0$ for $x>0$, then $f_{0}$ is everywhere conservative with respect to $f_{e}$.

Failure to identify the correct distribution underlying the Observer's performance results in biased estimates of $\beta_{o}$ that can mimic conservatism. The quantile mapping $\psi$ determines whether or not conservatism appears. If the relevant properties of $\psi$ can be computed from the Observer's performance, this potential source of apparent conservatism can be identified and then removed. One obvious place to look for information about $\psi$ is in the estimates $d_{e}$ of a fixed $d_{o}$, as $c_{o}$ varies.

From Eqs. (1) and (2), $d_{e}$ can be written as a function of $c_{o}\left(d_{o}\right.$ fixed):

$$
d_{e}=\psi\left(c_{o}\right)-\psi\left(c_{o}-d_{o}\right)
$$

which, after differentiating with respect to $c_{0}$ and changing variables, yields

$$
d_{e}^{\prime}=\psi^{\prime}(x+\Delta)-\psi^{\prime}(x-\Delta) .
$$

Comparing this last equation with Eq. (11), we see that, for fixed $d_{o}$, conservatism occurs precisely when

$$
d_{e}^{\prime}<0 \quad \text { iff } \quad c_{o}>\left(d_{o} / 2\right)
$$

But since $f_{e}$ and $f_{o}$ are even and unimodal, $\beta_{e}$ and $\beta_{o} \geqslant 1$ for $c_{o} \geqslant\left(d_{o} / 2\right)$.

THEOREM 4.9. The distribution $f_{o}$ is everywhere conservative with respect to the distribution $f_{e}$ iff, as $\beta_{o}$ varies, the estimate, $d_{e}$, of a fixed $d_{o}$ is a maximum when $\beta_{e}=\beta_{o}=1$, and decreases monotonically on both sides of this maximum.

The slight bow in Fig. 3b and 3d illustrates Eq. (14) and this theorem. In principle, the systematic variation in $d_{e}$ as $\beta_{o}$ varies is perfectly diagnostic of the conservatism that is introduced by a failure to choose the proper distribution for the Observer. These figures also suggest that the variation in $d_{e}$ may be slight and correspondingly difficult to detect in the data.

Further, to vary $\beta_{o}$ in practice requires altering the conditions of the experiment (vary payoffs, prior odds). It is commonly assumed that such manipulations do not 
affect $d_{o}$ but perhaps they do. Any systematic change in $d_{e}$, then, may diagnose a failure of the distributional assumptions-or may diagnose a failure of the assumption that $d_{o}$ is independent of payoffs, etc.

The conditions derived from Theorems 4.7 and 4.9 can be readily modified to predict what we called radicalism above, i.e., pairs of distributions, $f_{o}$ and $f_{e}$, where the fitted $\beta_{e}$ is consistently more extreme than the true $\beta_{o}$. A cursory examination of several commonly encountered distributions (e.g., the Laplace, Luce, and Cauchy distributions) discloses that all are conservative with respect to the Gaussian over the range of parameter values examined. Analogues of Theorems 4.7 and 4.9 of the previous section are easily proven for radicalism:

ThForfm 4.10. Assume that the quantile mapping, $\psi$, is twice differentiahle, i.e., that $\psi^{(2)}$ exists everywhere. Then $f_{o}$ is everywhere radical with respect to $f_{e}$ iff $\psi^{(2)}(x) \geqslant 0$ for $x>0$, with equality occurring on a set of Lebesgue measure 0 .

THEOREM 4.11. The distribution $f_{o}$ is everywhere radical with respect to the distribution $f_{e}$ iff, as $\beta_{o}$ varies, the estimate, $d_{e}$, of a fixed $d_{o}$ is a minimum when $\beta_{e}=\beta_{o}=1$, and increases monotonically on both sides of this minimum.

The results of this section are strong enough to permit computing a distribution that is everywhere conservative or everywhere radical with respect to any cumulative distribution function in S3. We need only pick a odd, increasing, continuous function $\psi$ with $\psi^{(2)}(x)<0$ for $x>0$. Since, by definition, $\psi=F_{e}^{-1} \cup F_{o}$, we have $F_{o}=F_{e} \circ \psi$. Applying Theorem 4.7 , we have shown

PROPOSITION 4.12. Let $F_{e}$ be any cdf in S3. Let $\psi$ be any odd monotone increasing function with $\psi^{(2)}(x)<0$ for $x>0$. Then $F_{o}=F_{e} \circ \psi$ is a cumulative distribution function that is everywhere conservative with respect to $F_{c}$. When $F_{e}$ is the Gaussian cumulative distribution function, denoted $\Phi$, then $F_{o}=\Phi \circ \psi$ is a cumulative distribution function that is everywhere conservative with respect to the Gaussian. $\psi(x)=x^{1 / 3}$ satisfies the proposition, and therefore $\Phi\left(x^{1 / 3}\right)$ is a distribution everywhere conservative with respect to the Gaussian.

The implication of the proposition is that, from the experimenter's point of view, there exist an infinitude of possible Observers $F_{o}$ that are optimal but appear conservative, as many as there are strictly increasing, odd functions concave on the positive reals. The example of the previous section is not an isolated property of the Laplace distribution. Given the results above, we can manufacture any number of examples by choice of $\psi$.

Similarly, to create a distribution that is everywhere radical with respect to the Gaussian, we need only pick a odd, continuous function $\psi$ with $\psi^{(2)}(x)>0$ for $x>0$ such as $x^{3}$. 


\section{A Necessary Condition for Conservatism/Radicalism}

Overview. In this section, we obtain a simple necessary condition for apparent conservatism based on the curvature at 0 of the two distributions. This condition permits rapid screening of distributions for possible radicalism/conservatism and allows us to exhibit a distribution that is locally radical with respect to the Gaussian. It also gives some insight into which distributions are not conservative with respect to the Gaussian. The conditions on $\psi$ above are currently the only necessary and sufficient conditions for radicalism/conservatism.

We also demonstrate that an index of the curvature of $f_{o}$ at 0 must be greater than that of $f_{e}$ if $f_{o}$ is to be everywhere apparently conservative with respect to $f_{e}$. The reverse inequality is a necessary condition for radicalism. As above, we are concerned with location TSDs for which the pdf's are drawn from S3. In addition, we now assume that the second derivatives, $f_{*}^{(2)}$, are defined in a neighborhood of 0 .

The index we use is

$$
\kappa_{0}(f)=\frac{\left|f^{(2)}(0)\right|}{f(0)^{3}}
$$

an inverse measure of the "flatness" of the pdf, $f(x)$, near 0 . A low value of $\kappa_{0}(f)$ indicates that $f(x)$ is relatively flat near its mode, and a high value of $\kappa_{0}(f)$ indicates that $f(x)$ is sharply peaked. We continue the notation of the previous section.

Suppose that the Observer sets the decision criterion $c_{o}$ at the median of the (symmetric) signal plus noise distribution. Then $c_{o}=d_{o}, p_{\mathrm{FA}}$ is $1-F_{o}\left(d_{o}\right)$, and the hit rate $p_{\mathbf{H}}$ is $\mathrm{p.5}$. The likelihood ratio at criterion is

$$
\beta_{o}=\frac{f_{o}(0)}{f_{o}\left(d_{o}\right)} .
$$

The Observer's responses are fitted by assuming a second even distribution $f_{e}$. By symmetry, the decision criterion $c_{e}$ must be placed at the median of the signal distribution since $p_{\mathbf{H}}=0.5$; also, the estimated likelihood at criterion would be

$$
\beta_{e}=\frac{f_{e}(0)}{f_{e}\left(c_{e}\right)}
$$

Since the modes of $f_{*}$ are at 0 , both likelihood criteria, $\beta_{*}$, exceed 1 , and conservatism would not be observed if $\beta_{e}>\beta_{o}$. Let

$$
y_{e}\left(d_{o}\right)=\frac{f_{e}\left(c_{e}\left(d_{o}\right)\right)}{f_{e}(0)},
$$

the reciprocal of $\beta_{e}$. (Note that we have made explicit the dependence of $c_{e}$ on $d_{o}$.) 
Let

$$
y_{o}\left(d_{o}\right)=\frac{f_{o}\left(d_{o}\right)}{f_{o}(0)},
$$

i.e., the reciprocal of $\beta_{o}$. Since zero is a mode of both distributions, $f_{*}, y_{*}^{(2)}$ are both negative at 0 . Further $y_{*}(0)=1, y_{*}^{\prime}(0)=0$, and

$$
\begin{aligned}
y_{o}^{(2)}(0) & =\frac{f_{o}^{(2)}(0)}{f_{o}(0)}, \\
y_{c}^{(2)}(0) & =\frac{f_{e}^{(2)}(0)\left[f_{o}(0)\right]^{2}}{\left[f_{e}(0)\right]^{3}} .
\end{aligned}
$$

Consequently, $y_{e}$ would be less than $y_{o}$ for some range of $d$ about 0 , if $y_{e}^{(2)}(0)<$ $y_{o}^{(2)}(0) \leqslant 0$. This last inequality implies (substituting from Eqs. (18) and (19)):

$$
\frac{f_{e}^{(2)}(0)}{\left[f_{e}(0)\right]^{3}}<\frac{f_{o}^{(2)}(0)}{\left[f_{o}(0)\right]^{3}}
$$

that is,

$$
\kappa_{0}\left(f_{e}\right)>\kappa_{0}\left(f_{o}\right)
$$

We have proven

THEOREM 5.1. If $\kappa_{0}$ for the true model is less than $\kappa_{0}$ for the fitted model, the estimated likelihood criterion would be more extreme than the true likelihood criterion for at least some $\left(p_{\mathrm{FA}}, p_{\mathrm{H}}\right)$ pairs.

Consequently,

COROLlaRY 5.2. If $f_{o}$ is everywhere conservative with respect to $f_{e}$, then $\kappa_{0}\left(f_{0}\right)>$ $\kappa_{0}\left(f_{e}\right)$, a necessary condition for conservatism.

EXAMPLES. Let $f_{e}$ be the unit-variance Gaussian distribution. It can be shown that the value of $\kappa_{0}$ for the Gaussian is $2 \pi$. $\kappa_{0}$ for the Laplace distribution discussed previously is $\infty>2 \pi$, consistent with our conclusion above that the Laplace distribution is apparently conservative with respect to the Gaussian. $\kappa_{0}$ for the Logistic distribution is 8 and for the Cauchy distribution is $2 \pi^{2}$. Hence these two distributions cannot be everywhere radical with respect to the Gaussian. Analyses similar to those performed for the Laplace distribution (not reported here) indicate that the Logistic and Cauchy are conservative with respect to the Gaussian. We next use the $\kappa_{0}$ criterion to provide an example of a pdf that is radical with respect to the Gaussian over at least part of its range.

The pdf, $f_{o}(x)=A \exp \left[-(|x| / B)^{3}\right], A \approx 0.315, B \approx 1.68$, which we call the hyper- 


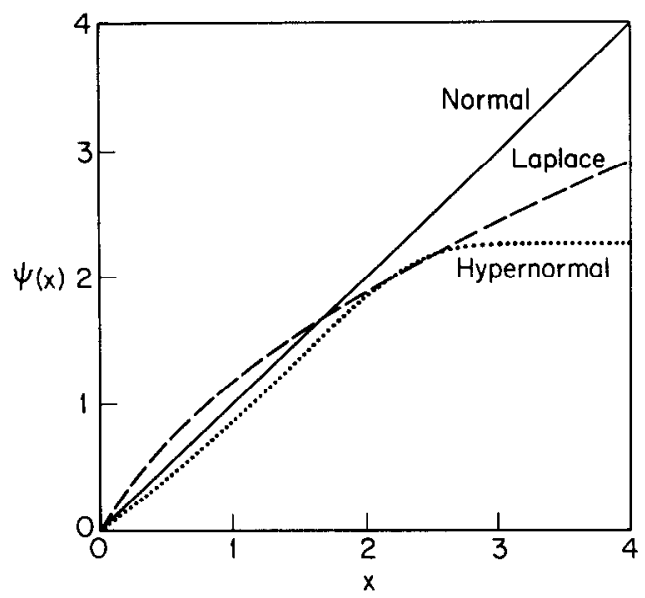

Fig. 5. Plots of the quantile functions $\psi$ between three distributions (Gaussian, Laplacian, and hypernormal) and the Gaussian distribution. See text for details.

normal distribution, has a mean of 0 and a variance of 1 . For this pdf, $\kappa_{0}$ is 0 , implying that this pdf would not be everywhere conservative with respect to the Gaussian pdf. Indeed, Fig. $3 \mathrm{e}$ shows the radicalism of the distribution $f_{o}$ with respect to the distribution $f_{e}$ for this choice of distributions. Figure $3 f$ shows that the estimate, $d_{e}$, has a local minimum when $\beta_{o}=1$, consistent with Theorem 2.

For the purpose of comparison, we have plotted in Fig. 5 the quantile mapping, $\psi$, for $f_{o}$ Gaussian, Laplacian, and hypernormal, and $f_{e}$ Gaussian. As expected from Theorem 1, the first function is linear, the second is concave, and the third is not concave for all $x$. Other calculations show that when, for example, $f_{e}$ is the Cauchy pdf and $f_{o}$ is, in turn, Gaussian, Laplacian, and hypernormal, $\psi$ is always convex increasing, suggesting that these choices of $f_{o}$ are radical with respect to the Cauchy pdf. This convexity of $\psi$, coupled with the fact that $\kappa_{0}$ is greater for the Laplacian pdf than for the Cauchy pdf, demonstrates that the above condition on $\kappa_{0}$ is not sufficient even for local conservatism. The condition on $\kappa_{0}$ is necessary for $\psi$ to be concave as we prove below. The usefulness of this condition stems from the fact that the shape of the pdf is more easily interpretable than the curvature of $\psi$. So far we have been unable to obtain a sufficient condition that is as easily interpretable as the condition on $\kappa_{0}$.

Proposition 5.3. If $\psi$ is concave, then $\kappa_{0}\left(f_{e}\right)>\kappa_{0}\left(f_{o}\right)$.

Proof. From the definition of $\psi, F_{e} \circ \psi=F_{o}$. On differentiating this equation twice, we get $\psi^{\prime}(x)=f_{o}(x) / f_{e} \circ \psi(x)$, and

$$
\psi^{(2)}(x)=\frac{f_{o}(x)^{2}}{f_{e} \circ \psi(x)}\left[\frac{f_{o}^{\prime}(x)}{f_{o}(x)^{2}}-\frac{f_{e}^{\prime}(x)}{f_{e}(x)^{2}}\right] .
$$


Since 0 is the median of the pdf's, $\psi(0)=0$. Therefore, for $x$ close to 0 ,

$$
\psi(x) \approx \psi^{\prime}(0) x=x \frac{f_{o}(0)}{f_{e}(0)} .
$$

Also, since $f_{*}$ are even and unimodal, $f_{*}^{\prime}(0)=0$, and $f^{(2)}(0) \leqslant 0$. Now $\psi^{(2)}<0$ implies that

$$
\frac{f_{o}^{\prime}(x)}{f_{o}(x)^{2}}<\frac{f_{e}^{\prime}(x)}{f_{e}(x)^{2}}
$$

On expanding each side of the inequality in a Taylor series about $x=0$, substituting for $\psi(x)$, and $f_{*}^{\prime}(0)$, and noting that $f_{*}^{(2)} \leqslant 0$, we get

$$
\kappa_{0}\left(f_{o}\right)>\kappa_{0}\left(f_{e}\right)
$$

as required.

\section{Distributional INFORMATION IN THE OBSERVER'S ISOSENSITIVITY CURVES}

In the preceding sections, attention is confined to the subset $\mathbf{S 3}$ of the full set of location TSDs. We now drop that restriction and consider all location TSDs.

Biases introduced by incorrectly specifying the Observer's underlying distribution have led us to examine what can be inferred about the Observer's underlying distribution from detection data. It is often stated or implied that an isosensitivity curve completely characterizes the Observer's performance. (See, for example, Coombs, Dawes, \& Tversky, 1970, p. 175). We believe that the meaning of this statement is that, once a given $f_{o}$ is assumed, the isosensitivity curve is uniquely determined by $d_{o}$. Each isosensitivity curve corresponds to exactly one value of $d_{o}$. However, one can inquire after a deeper meaning to the statement and ask, for example, "What information about the underlying pdf $f_{o}$ is available in the subject's performance as summarized by the isosensitivity curve?"

It is known that a given isosensitivity curve can be generated by infinitely many pairs of stimulus distributions, $F_{\mathrm{N}}(x)$, and $F_{\mathrm{SN}}(x)$ (Laming, 1973, pp. 100-101; Marley, 1971). Marley (1971) also provides necessary conditions for a set of isosensitivity curves to be generated by a location TSD. In this section, we consider the simpler question of whether two distinct location TSDs can generate the same set of isosensitivity curves. The stimulus distributions are not assumed to be even (symmetric about the median). We show, after some intermediate results, that, given two isosensitivity contours, there is at most one location TSD that could generate them both. We also show that the isosensitivity contour generated by a given location TSD can be generated also by at least one other location TSD. This last result implies that the minimum number of isosensitivity contours required for the characterization of a location TSD is two. 
Levine (1970) provides necessary and sufficient conditions for the existence of transformations that render curves parallel. Location TSDs have cumulative distribution functions that are parallel curves in Levine's sense and the requirement that there be only one location TSD generating a given set of isosensitivity curves can be construed withing Levine's framework as an assertion concerning the uniqueness of the transformation rendering certain cumulative distribution functions parallel. Levine's necessary and sufficient conditions must, of course, be equivalent to the necessary and sufficient conditions we derive below, but we have found no direct path from his result to ours. Since Levine's results may extend to the location-scale TSD case discussed in the dnext section, while our results, so far, cannot, it would be desirable to express Levine's results in terms appropriate to TSD.

An isosensitivity curve for a location TSD is determined by specifying the underlying distribution, $f_{1}$, and the location parameter, $d_{1}$, separating the noise and signal plus noise distributions. Each valuc of $d_{1}$ generates a distinct isosensitivity curve. Let us denote the set of isosensitivity curves generated by a particular location TSD based on $f_{1}$ as $\mathbf{I}\left(f_{1}\right)$. Let $f_{2}$ be a second distribution such that $\mathbf{I}\left(f_{1}\right)=$ $\mathbf{I}\left(f_{2}\right)$. That is, for any isosensitivity curve generated by $f_{1}$ with location parameter $d_{1}$, there is $d_{2}$ such that $f_{2}$ with location parameter $d_{2}$ sweeps out the same isosensitivity curve. Let $F_{1}$ and $F_{2}$ be the cdfs of $f_{1}$ and $f_{2}$, respectively, and suppose that both distributions have been normalized to have a median of 0 and a semiinterquartile range of 1 . We next show that $f_{1}$ and $f_{2}$ are identical: The full set of isosensitivity curves produced from a location TSD determines the underlying family distribution.

\section{THEOREM 6.1. $\mathbf{I}\left(f_{1}\right)=\mathbf{I}\left(f_{2}\right)$ iff $f_{1}=f_{2}$.}

Proof. The isosensitivity curves can be ordered monotonically with respect to $d_{1}$ (for the location TSD based on $f_{1}$ ) and with respect to $d_{2}$ (for the location TSD based on $f_{2}$ ). Therefore, two distinct values of $d_{1}$ correspond to distinct isosensitivity curves and, therefore, to distinct values of $d_{2}$. This shows that $d_{2}$ is a welldefined function of $d_{1}$. But these two variables can be interchanged in the argument to show that $d_{1}$ is a continuous surjective (onto) function of $d_{2}$. This implies that there is a monotonic function $m$ such that $d_{2}=m\left(d_{1}\right)$. Then Eqs. (1) and (2) imply

$$
\psi\left(x-d_{1}\right)=\psi(x)-m\left(d_{1}\right),
$$

where $\psi=F_{2}^{-1} \circ F_{1}$. Setting $d_{1}=0$ in the previous equation gives us $m(0)=0$, and it can be shown that $\psi(0)=0$. On putting $x=0$ in Eq. (22), we have

$$
\psi\left(d_{1}\right)=-m\left(-d_{1}\right),
$$

so that $m(x)=-\psi(-x)$. Therefore, substituting $y=-d_{1}$ and substituting for $m$ in Eq. (22), we obtain

$$
\psi(x+y)=\psi(x)+\psi(y)
$$


and, consequently, $\psi(x)=u x$ for some constant $u$ (Aczel, 1966, pp. 31, 32). Since $f_{1}$ and $f_{2}$ are normalized to have the same semi-interquartile range, $u=1$ (shown above), $\psi$ is the identity, and $f_{1} \equiv f_{2}$ as desired.

This result can be strengthened considerably. Next we demonstrate that as few as two isosensitivity curves, corresponding to appropriate choices of the parameter $d$ for a location TSD Observer, completely specify that Observer. (In the next section, we demonstrate that three isosensitivity curves suffice to determine a particular type of location-scale TSD Observer.) In practice, then, a small number of isosensitivity curves, corresponding to different location parameters $d_{o}$ for a TSD Observer, determine the Observer. All location TSDs share the degenerate isosensitivity contour corresponding to $d=0$. For convenience in the presentation below we ignore this contour: "isosensitivity contour" means "non-degenerate isosensitivity contour."

Also, in stating the theorems below, it is convenient for us to refer to the zero function as a "periodic function."

First we prove a simple lemma.

LEMMA 6.2. Let $g$ be a real-valued function, and suppose $g(x+a)=g(x)+b$, for all $x$, where $a$ and $b$ are constants. Then $g$ is the sum of a linear function, $g_{\mathrm{L}}$, and a periodic function, $g_{\mathrm{P}}$, either of which may be zero. Further, if $h_{\mathrm{L}}$ and $h_{\mathrm{P}}$ are a linear and a periodic function that also sum to $g$, then $h_{\mathrm{L}}=g_{\mathrm{L}}$ and $h_{\mathrm{P}}=g_{\mathrm{P}}$ : the decomposition is unique.

Proof. Aczel proves this result in his discussion of Abel's Equation (Aczel, 1966). Let

$$
\begin{aligned}
& g_{\mathrm{L}}(x)=\frac{b}{a} x+g(0), \\
& g_{\mathrm{P}}(x)=g(x)-g_{\mathrm{L}}(x) .
\end{aligned}
$$

Then $g_{\mathrm{P}}$ is either periodic, with $a$ being a multiple of the period, or zero, because

$$
g_{\mathrm{P}}(x+a)=g(x+a)-g_{\mathrm{L}}(x+a)=g_{\mathrm{P}}(x) .
$$

To prove uniqueness, one can evaluate $g_{\mathrm{L}}+g_{\mathrm{P}}$ and $h_{\mathrm{L}}+h_{\mathrm{P}}$ at $a$ and $2 a$. Both periodic functions vanish, $g_{\mathrm{L}}=h_{\mathrm{L}}$ necessarily, and uniqueness follows.

Now suppose that there are two isosensitivity curves that are generated by (a) the sensitivity parameter values $d_{1}$ and $d_{1}^{\prime}$ within the location TSD, $f_{1}$, and also by (b) the sensitivity parameter values $d_{2}$ and $d_{2}^{\prime}$ within the location TSD, $f_{2}$. Let $c_{1}$ and $c_{2}$ denote criterion values within the models $f_{1}$ and $f_{2}$, respectively.

Let $F_{1}$ and $F_{2}$ be the cdf's corresponding to $f_{1}$ and $f_{2}$, and let $\psi=F_{2}^{-1} \circ F_{1}$ be the quantile function mapping $f_{1}$ to $f_{2}$, where it is assumed that the distributions have been normalized to have median 0 and semi-interquartile range 1 . We denote by $\mathrm{I}[f, d]$ the isosensitivity curve within a location TSD, $f$, generated by the sensitivity parameter $d$. 
Definition 6.3. For two positive real numbers, $x$ and $y$, we say that $x$ and $y$ are relatively prime if there is no real $z$ with $x / z$ and $y / z$ both integers.

Some illustrations of relative primeness: The real numbers 3.2 and 1.6 are not relatively prime; the numbers 1 and $\pi$ are. If two numbers are picked independently at random from the uniform distribution on $[0,1]$, they will be relatively prime with probability 1 . If two numbers are picked from any smooth joint distribution on $\mathbf{R}^{2}$, the real plane, they will be relatively prime with probability 1 .

Next we show that, if two location TSDs share two isosensitivity curves, then the two TSDs are identical.

THEOREM 6.4. If $\mathbf{I}\left[f_{1}, d_{1}\right]=\mathbf{I}\left[f_{2}, d_{2}\right]$ and $\mathbf{I}\left[f_{1}, d_{1}^{\prime}\right]=\mathbf{I}\left[f_{2}, d_{2}^{\prime}\right]$, where $d_{1}$ and $d_{1}^{\prime}$ are relatively prime, then $f_{1} \equiv f_{2}$.

Proof. As in an earlier section, it can be shown, for the first isosensitivity curve, that

$$
\psi\left(c_{1}\right)=c_{2}
$$

and

$$
\psi\left(c_{1}-d_{1}\right)=c_{2}-d_{2}
$$

so that

$$
\psi\left(c_{1}-d_{1}\right)=\psi\left(c_{1}\right)-d_{2}
$$

Applying the lemma, we see that $\psi=\psi_{\mathrm{L}}+\psi_{\mathrm{P}}$, where either $\psi_{\mathrm{P}}$ is zero or $d_{1}$ is an integral multiple of its period. Repeating the above construction for the second isosensitivity curve, we conclude that either $\psi_{\mathrm{P}}$ is zero or else both $d_{1}$ and $d_{1}^{\prime}$ are integral multiples of its period. Since $d_{1}$ and $d_{1}^{\prime}$ are relative prime, $\psi_{\mathrm{P}}$ must be zero and $\psi$ must be a linear transformation. Since $f_{1}$ and $f_{2}$ are noth normalized, $\psi$ must be the identity: $f_{1}=f_{2}$ as desired.

As part of the proof of Theorem 5, we proved a result required for the next theorem.

LEMMA 6.5. If $\mathbf{I}\left[f_{1}, d_{1}\right]=\mathbf{I}\left[f_{2}, d_{2}\right]$, then the quantile map $\psi$ relating $f_{1}$ and $f_{2}$ can be decomposed into the sum of a linear and a periodic map of period $d_{1}$.

We next show that the number of isosensitivity curves specified in the conditions of Theorem 6.4 cannot be reduced to one: There are isosensitivity curves generated by two distinct location TSDs.

THEOREM 6.6. There are distinct location TSDs generating the same isosensitivity contours.

Proof. We need only construct distinct distributions $f_{1}, f_{2}$, with parameters $d_{1}, d_{2}$, respectively, having the same isosensitivity curve: $\mathbf{I}\left[f_{1}, d_{1}\right]=\mathbf{I}\left[f_{2}, d_{2}\right]$. Let 
$\psi(x)=x+(1 / 4 \pi) \sin (2 \pi x)$. Note that $\psi$ is odd, continuous, and increasing. $\psi$ is also the sum of a linear and a periodic function of period 1 . For any normalized $F_{2}$, let $F_{1}=F_{2} \circ \psi$. Let $d_{1}=d_{2}=1$. Note that $F_{1}$ is also normalized. The two distributions are distinct as both are normalized yet $F_{1}(1 / 4) \neq F_{2}(1 / 4)$.

Now we want to show that if $\left(p_{\mathrm{FA}}, p_{\mathrm{H}}\right)$ is on the isosensitivity curve $\mathrm{I}\left[f_{1}, d_{1}\right]$, then it is also on the isosensitivity curve $\mathrm{I}\left[f_{2}, d_{2}\right]$ and conversely. If $\left(p_{\mathrm{FA}}, p_{\mathrm{H}}\right)$ is on the isosensitivity curve $\mathbf{I}\left[f_{1}, d_{1}\right]$, then there is an $x$ with

$$
F_{1}(x)=1-p_{\mathrm{FA}}
$$

and

$$
F_{1}(x-1)=1-p_{\mathrm{H}} .
$$

But then, substituting $F_{1}=F_{2} \circ \psi$,

$$
F_{2}(\psi(x))=1-p_{\mathrm{FA}}
$$

and

$$
F_{2}(\psi(x-1))=1-p_{\mathrm{H}} .
$$

Since $\psi(x-1)=\psi(x)-1$, the second equation becomes

$$
F_{2}(\psi(x)-1)=1-p_{\mathrm{H}},
$$

and, substituting $y=\psi(x)$, we see that $\left(p_{\mathrm{FA}}, p_{\mathrm{H}}\right)$ is on the isosensitity curve I $\left[f_{2}, d_{2}\right]$. Since $\psi$ is invertible, we can reverse the roles of the two distributions and prove that every point on $\mathbf{I}\left[f_{2}, d_{2}\right]$ is on $\mathbf{I}\left[f_{1}, d_{1}\right]$ as well. The two isosensitivity curves coincide, yet $f_{1}$ is distinct from $f_{2}$.

The construction used in the previous theorem proves the stronger result,

THEOREM 6.7. If $\mathbf{I}$ is an isosensitivity curve generated by a location TSD, then there are infinitely many location TSDs that also generate the contour $\mathbf{I}$.

Proof. The $\psi$ used above may be used to generate a second location TSD. There is a distinct location TSD for each of the infinitely many possible choices of $\psi$ that is the sum of a linear and periodic function of period 1 , that is odd and monotone increasing, and that carries normalized distributions into normalized distributions.

\section{An Extension to Location-Scale TSDs}

The class of location TSDs is a generalization of equal-variance Gaussian TSD relaxing the assumptions on the form of the underlying distributions. We can 
similarly generalize unequal-variance Gaussian TSD to the class of location-scale $T S D s$ defined by the requirement that

$$
F_{\mathrm{SN}}(x)=F_{\mathrm{N}}\left(\frac{x-d}{s}\right),
$$

where $d$ is as before a location parameter and $s$ is a scaling parameter.

When the range of permissible TSDs is expanded to include a particular type of location-scale TSDs with possibly asymmetric distributions, a result analogous to the previous theorem can be proved given three isosensitivity curves. Let $d_{1}^{\prime \prime}$, etc., denote the parameters corresponding to the third isosensitivity curve; otherwise the notation is as in the previous theorem. $f_{1}$ and $f_{2}$ are now location-scale TSDs with fixed scale parameters $s_{1}$ and $s_{2}$. It should be noted that the more typical locationscale assumption is that the scale parameter varies with separation between the stimulus distributions. However, we have not had much success in studying this larger class of location-scale TSDs. The next result concerns a location-scale model that is, therefore, more restrictive than is generally assumed for location-scale models.

THEOREM 7.1. Suppose that

$$
\mathbf{I}\left[f_{1}, s_{1}, d_{1}^{(i)}\right]=\mathbf{I}\left[f_{2}, s_{2}, d_{2}^{(i)}\right], \quad i=1,2,3,
$$

where $d_{1}^{(2)}-d_{1}^{(1)}$ and $d_{1}^{(3)}-d_{1}^{(1)}$ are relatively prime. Then $f_{1} \equiv f_{2}$.

Proof. For the location-scale case,

$$
\begin{gathered}
\psi\left(c_{1}^{(1)}\right)=c_{2}^{(1)}, \\
\psi\left(\frac{c_{1}^{(1)}-d_{1}^{(1)}}{s_{1}}\right)=\frac{c_{2}^{(1)}-d_{2}^{(1)}}{s_{2}},
\end{gathered}
$$

for the first isosensitivity curve. Consequently,

$$
s_{2} \psi\left(\frac{x^{(1)}}{s_{1}}\right)=\psi\left(x^{(1)}+d_{1}^{(1)}\right)-d_{2}^{(1)},
$$

where $x^{(1)}=c_{1}^{(1)}-d_{1}^{(1)}$. For the second isosensitivity curve we obtain an analogous equation in $x^{(2)}=c_{1}^{(2)}-d_{1}^{(2)}$. By varying $c_{1}^{(2)}$ we can set $x^{(1)}=x^{(2)}=x$, so that

$$
s_{2} \psi\left(\frac{x}{s_{1}}\right)=\psi\left(x+d_{1}^{\prime}\right)-d_{2}^{\prime} .
$$

Then, equating (33) and (34) and setting $z=x+d_{1}^{(1)}, a=d_{1}^{(2)}-d_{1}^{(1)}$, and $b=$ $d_{2}^{(2)}-d_{2}^{(1)}$, we have,

$$
\psi(z)=\psi(z+a)+b
$$

The remainder of the proof involves retracing the above steps for the first and third 
isosensitivity curves, obtaining a second equation involving $\psi$ analogous to (35), and then applying the lemma as in the previous theorem.

Levine $(1970,1972)$ examines the groups of transformations rendering curves parallel or rendering curves into a common shape. As noted above, the problem of the uniqueness of the location TSD generating a given set of isosensitivity curves can be expressed in Levine's framework as an assertion concerning the uniqueness of a specific transformation. It may be possible also to state the problem of the uniqueness of the location-scale TSD that generates a given set of isosensitivity curves within this same framework, but we have been unable to do so.

\section{Concluding Remarks}

The model of the Observer in a Yes-No detection task provided by the theory of signal detectability consists of three functions: (a) the distribution of the sensory information $X$ available to the Observer on trials where the signal is absent $\left(F_{\mathrm{N}}\right)$, (b) the distribution of $X$ on trials where the signal is present $\left(F_{\mathrm{SN}}\right)$, and (c) a decision rule $D(\cdot)$ that, when applied to $X$ on a given trial, produces a Yes or No response. The experimenter's task is to determine these three functions $\left(F_{\mathrm{N}}, F_{\mathrm{SN}}, D\right)$, given the Observer's hit and false alarm rates. Without some further restriction on distributions and decision rules, the experimenter cannot succecd in identifying the Observer from the data available.

In practice, then, the missing information specifying the Observer is assumed. In the most familiar case, $F_{\mathrm{N}}$ is assumed to be Gaussian with mean 0 and unit variance, and $F_{\mathrm{SN}}$ is assumed to be Gaussian with unknown mean $d^{\prime}$ and unit variance. The decision rule $D$ is taken to be Yes iff $X>c$, in which the only unknown is the criterion parameter $c$. The analytical task is to estimate the free parameters remaining in the model of the Observer and use these as indices of the Observer's performance. In particular, estimates of the critcrion for human Observers that are less extreme than the value that is optimal for the decision are taken as evidence that human Observers behave conservatively.

The predominant response in the literature to this finding of suboptimal behavior has been a search for heuristics accounting for the behavior. In contrast, the concern in this paper has been to question whether the "finding" might be due to suboptimal model-fitting by the experimenter, rather than suboptimal behavior by the Observer. ${ }^{1}$ We have found this to be the case. A discrepancy between assumed and truc sensory distributions can result in labelling as conservative behavior that is in fact optimal. The appearance of conservatism is governed by the curvature of the function mapping the quantiles of the true distribution into the quantiles of the assumed distribution.

${ }^{1}$ As noted above, findings of suboptimality in human judgment and decision-making also have prompted an examination of whether suboptimality inheres in the model-fitting rather than in the Observer's behavior. For example, Kahneman et al. (1982) suggest that the model of probability theory used by the Observer may be different from the classical model that is typically used by the experimenter. 
This result led us to investigate ways in which distributional information might be extracted from the Observer's responses. In the weak form of this investigation, we looked for indications within the data that particular distributional assumptions might be in error. We showed that slight, but systematic variations in the estimate of sensitivity, as the Observer varies $c$, can be adequately diagnostic of an incorrect distributional assumption. In a similar vein, it is not difficult to show that there are slight, but systematic changes in the shape of the isosensitivity curve as we go from one family of location TSDs to another (Laming, 1973, pp. 122-124). For example, the slope of the isosensitivity curve near the origin depends on the distribution: it is 1 for the Cauchy, finite and greater than 1 for the Laplacian, and infinite for the Gaussian (see Fig. 4). However, since the estimation of the slope of the isosensitivity curve in this region is unreliable, it would be difficult to use this feature of the curve to test the adequacy of a distributional assumption. (Swets (1986a) discusses the problems of diagnosing distributional failures.)

The strong form of our investigation of distributional information focused on the isosensitivity curve and resulted in Theorems 4 and 5 . These results show that, in location TSD models, distributional information is fully contained in the isosensitivity curve, and that, in principle, no further assumptions about the Observer's underlying distributions are necessary. Rather surprisingly, the data summarized in as few as two isosensitivity curves from a location TSD specify the underlying distributions completely.

In practice, it is unlikely that distributional information can be extracted from an isosensitivity curve in a transparent or tractable form until a tractable (nonparametric) representation of the isosensitivity curve (or, equivalently, of the sensory distributions) is posited. However, our results suggest that there is a cost associated with constraining the set of possible isosensitivity curves too severely (for example, to the parametric Gaussian or Laplace families of curves). An intermediate approach would be a semi-parametric one (Bloxom, 1985) in which an isosensitivity curve is characterized by (a) a parameter specific to that curve and (b) a function that is the same for all isosensitivity curves but not specified a priori. This invariant function could be drawn from a prespecified class of splines. Then, given a set of empirical isosensitivity curves and the invariant function, the parameters for the curves could be estimated by, e.g., methods used by Bloxom (1985). It is likely that this approach would reduce significantly the bias in the criterion estimates, and further work in this direction seems warranted.

\section{REFERENCES}

ACZEL, J. (1966). Lectures on functional equations and their applications. New York: Academic Press.

Altham, P. M. E. (1973). A non-parametric measure of signal discriminability. British Journal of Mathematical and Statistical Psychology, 26, 1-12.

Apostol, T. M. (1967). Calculus (Vol. I). One-variable calculus with an introduction to linear algebra (2nd ed.). Lexington, MA: Xerox.

BLoxom, B. (1985). Considerations in psychometric modeling of response time. Psychometrika, 50, 383-397. 
COOMBS, C. H., DAWES, R. M. \& TVERSKy. A. (1970). Mathematical psychology: an elementary introduction. Englewood Cliffs, NJ: Prentice-Hall.

Creelman, C. D., \& Donaldson, W. (1968). ROC curves for discrimination of linear extent. Journal of Experimantal psychology, 77, 514-516.

Dorfman, D. D., Beavers, L. L., \& Saslow, C. (1973). Estimation of signal detection theory parameters from rating-method data: A comparision of the method of scoring and direct search. Bulletin of the Psychological Society, 1, 207-208.

Dusolr, A. E. (1974). Thomas and Legge's matching hypothesis for detection and recognition tasks: Two tests. Perception and Psychophysics, 16, 466-470.

Egan, J. P. (1975). Signal detection theory and ROC analysis. New York: Academic Press.

GNANADESIKAN, R.(1977). Methods for statistical analysis of multivariate observations. New York: Wiley.

GreEN, D. M., \& SwETs, J. A. (1966). Signal detection theory and psychophysics. New York: Wiley. Reprinted (1974) with corrections. Huntington, NY: Krieger. (References are to 1974 reprint)

GREY, D. R., \& MORGAN, B. J. T. (1972). Some aspects of ROC curve-fitting: Normal and logistic models. Journal of Mathematical Psychology, 9, 128-139.

Healy, A. F., \& Kubovy, M. (1981). Probability matching and the formation of conservative decision rules in a numerical analog of signal detection. Journal of Experimental Psychology: Human Learning and Memory, 7, 344-354.

Johnson, N. L., \& Kotz, S. (1970). Continuous univariate distributions-2. New York: Wiley.

Kahneman, D., Slovic, P., \& Tversky, A. (1982). Judgment under uncertainty: Heuristic biases. Cambridgc: Cambridgc univ. Press.

KuBovy, M. (1977). A possible basis for conservatism in signal detection and probabilistic categorization tasks. Perception and Psychophysics, 22, 277-281.

Laming, D. R. J. (1973). Mathematical psychology. New York: Academic Press.

LAming, D. R. J. (1986). Sensory analysis. London: Academic Press.

LEVINE, M. V. (1970). Transformations that render curves parallel. Journal of Mathematical Psychology, $7,410-443$.

LEVINE, M. V. (1972). Transforming curves into curves with the same shape. Journal of Mathematical Psychology, 9, 1-16.

Lockhart, R. S., \& Murdock, B. B. (1970). Memory and the theory of signal detection. Psychological Bulletin, 74, 100-109.

Marley, A. A. J. (1971). Conditions for the representation of absolute judgment and pair comparison isosensitivity curves by cumulative distributions. Journal of Mathematical Psychology, 8, 554-590.

Morgan, B. J. T. (1976). The uniform distribution in signal detection theory. British Journal of Mathematical and Statistical Psychology, 29, 81-88.

OGILVIE, J. C., \& CREelmaN, C. D. (1968). Maximum-likelihood estimation of receiver operating characteristic curve parameters. Journal of Mathematical Psychology, 5, 377-391.

PARKS, T. E. (1966). Signal-detectability theory of recognition memory performance. Psychological Review, 73, 44-58.

Poli.ACK, I., \& Hsifh, R. (1969). Sampling variability of the area under the ROC curve and of $d_{c}^{\prime}$. Psychological Bulletin, 71, 161-173.

SwETS, J. A. (1986a). Indices of discrimination or diagnostic accuracy: Their ROCs and implied models. Psychological Bulletin, 99, 100-117.

SwETS, J. A. (1986b). Form of empirical ROCs in discrimination and diagnostic tasks: Implications for theory and measurement of performance. Psychological Bulletin, 99, 181-198.

TANNER, W. P., JR., \& Swets, J. A. (1954). A decision-making theory of visual detection. Psychological Review, 61, 401-409.

Thomas, E. A. C. (1975). Criterion adjustment and probability matching. Perception and Psychophysics, $18,158-162$.

Thomas, E. A. C., \& LegGe, D. (1970). Probability matching as a basis for detection and recognition decision. Psychological Review, 77, 65-72.

ReCEIVED: August 4, 1989 